\title{
SYMMETRICAL JOUKOWSKY AIRFOILS IN SHEAR FLOW $\dagger$
}

\author{
BY \\ HSUE-SHEN TSIEN \\ California Institute of Technology
}

1. Problem. The usual two-dimensional theory of airfoils assumes a uniform velocity for points far from the airfoil. There are many applications where this condition is not satisfied. For example near the ground there is a large vertical velocity gradient, and therefore a first approximation to the problem can be obtained by assuming a linear velocity distribution. This has, in fact, been done by H. v. Sanden ${ }^{1}$ in connection with O. Lilienthal's experiments in natural wind. However, v. Sanden used a numerical method of integrating the differential equation and carried out the calculation only for a wedge-shaped body. Th. von Kármán* suggested to the author to take up this problem again in order to develop a more complete theory. Hence in the

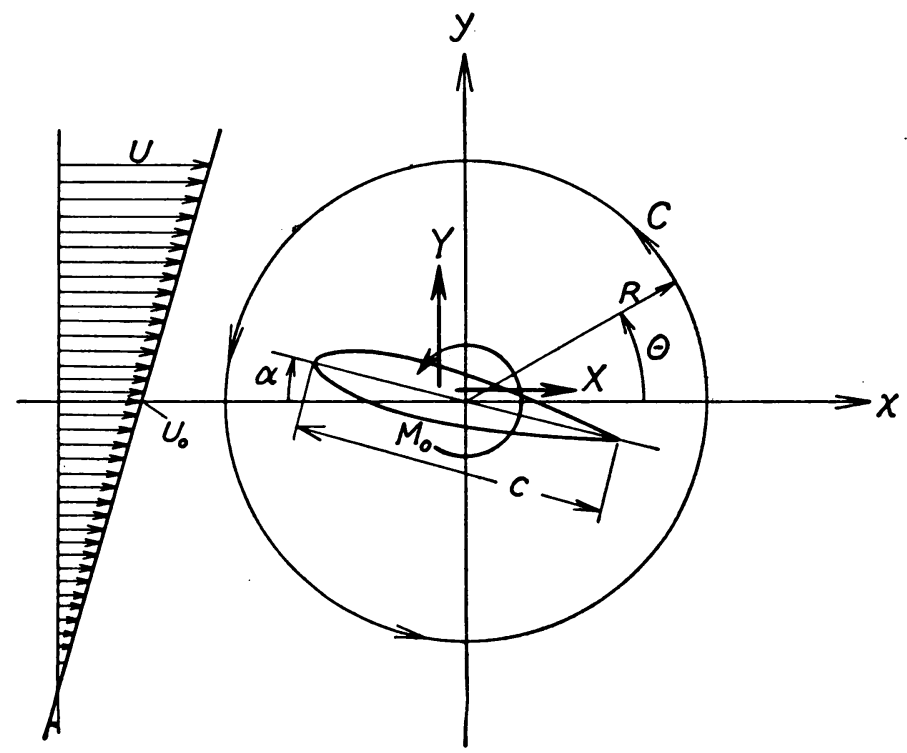

Fig. 1. Body in a Shear Flow.

† Received Dec. 27, 1942.

${ }^{1}$ v. Sanden, H., Über den Auftrieb im natürlichen Winde, Zeitschrift f. Math. u. Phys., 61, 225, (1912).

* The author wishes to thank Dr. von Kármán for suggesting the problem and his kind interest during the course of the work. 
first part of this paper, a generalization of the well-known Blasius theorem for calculating aerodynamic forces acting on an airfoil is given. Then the result is applied to the case of symmetrical Joukowsky airfoils and the final data are given in a number of tables and graphs.

2. Method of solution. By setting up the problem as shown in Fig. 1, the velocity distribution far from the airfoil is given by

$$
\begin{aligned}
& u=U_{0}\left(1+K \frac{y}{c}\right) \\
& v=0
\end{aligned}
$$

where $U_{0}$ is the undisturbed flow velocity along the $x$-axis and $K$ is the nondimensional velocity gradient of the undisturbed flow. $c$ is some dimension of the body immersed in the stream, e.g., the chord of the airfoil. Then the vorticity at locations far from the airfoil can be calculated from Eq. (1) as

$$
\frac{\partial v}{\partial x}-\frac{\partial u}{\partial y}=-U_{0} K / c
$$

which is a constant. However, in the flow of non-viscous incompressible fluid, the vorticity is associated with the fluid and maintains its strength. Consider the field of flow starting from the far left, where the vorticity is constant and equal to $-U_{0} K / c$. This value of vorticity is carried with the fluid over the whole field of flow. Therefore, the flow problem on hand is one with constant vorticity distribution.

To satisfy the equation of continuity,

$$
\frac{\partial u}{\partial x}+\frac{\partial v}{\partial y}=0
$$

the stream function $\psi$ is introduced. It is defined by

$$
u=\frac{\partial \psi}{\partial y}, \quad v=-\frac{\partial \psi}{\partial x} .
$$

$u, v$ are the components of velocity in the $x$-direction and the $y$-direction. Due to constant vorticity distribution, the vorticity equation is

$$
\frac{\partial v}{\partial x}-\frac{\partial u}{\partial y}=-U_{0} K / c .
$$

By using Eq. (4), Eq. (5) can be written as

$$
\frac{\partial^{2} \psi}{\partial x^{2}}+\frac{\partial^{2} \psi}{\partial y^{2}}=U_{0} K / c .
$$

Therefore the flow problem is reduced to that of solving Eq. (6). 
The stream function $\psi_{0}$ of the undisturbed flow given by Eq. (1) is

$$
\psi_{0}=U_{0}\left(y+\frac{K}{2} \frac{y^{2}}{c}\right)
$$

which can be easily verified by means of Eq. (4). The mathematical problem can be considerably simplified by introducing the stream function $\psi_{1}$ due to the presence of the body defined as

$$
\psi=\psi_{0}+\psi_{1} .
$$

By substituting this expression for $\psi$ into Eq. (6), the equation for $\psi_{1}$ is simply

$$
\frac{\partial^{2} \psi_{1}}{\partial x^{2}}+\frac{\partial^{2} \psi_{1}}{\partial y^{2}}=0
$$

This is the Laplace equation. Therefore any solution of the Laplace equation

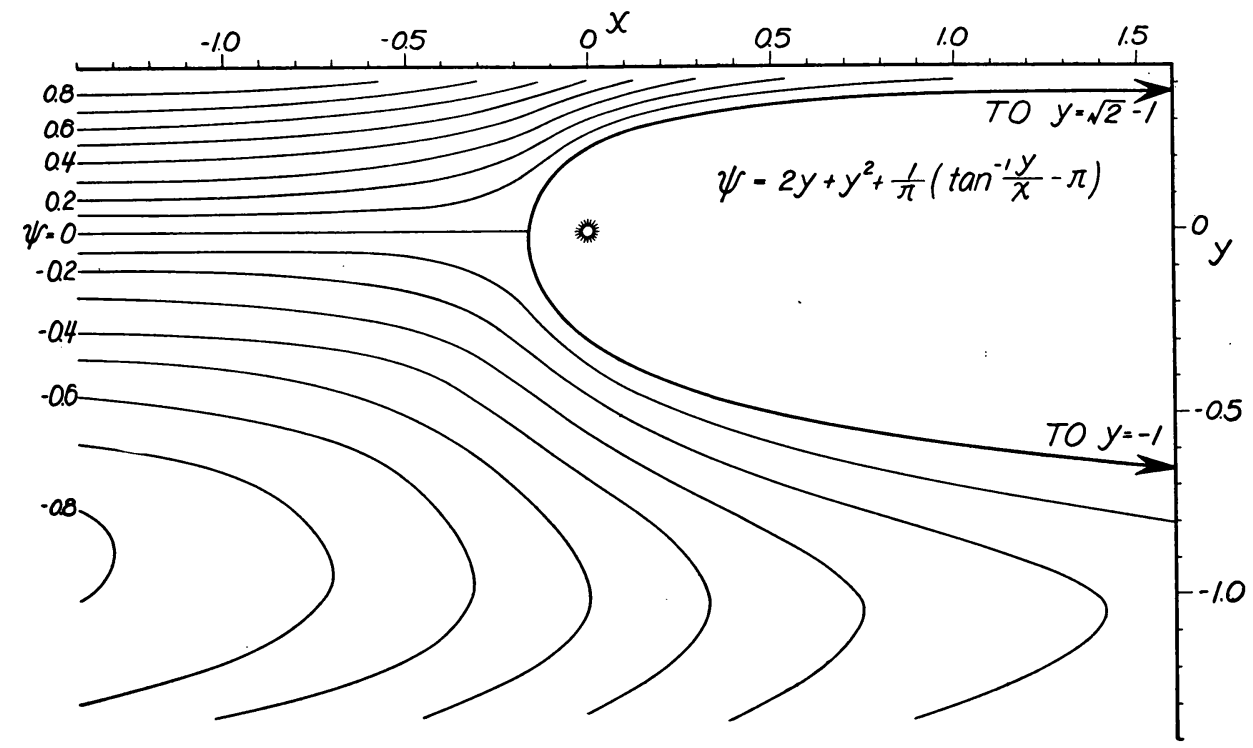

FIG. 2. Source in a Shear Flow.

combined with $\psi_{0}$ will satisfy Eq: (6). For example, we can combine $\psi_{0}$ with a source

or a vortex

$$
\begin{aligned}
& \psi_{1}=U_{0} b_{0} \theta \\
& \psi_{1}=U_{0} a_{0} \log r
\end{aligned}
$$

as shown in Figs. 2, 3, and 4. Here

$$
\theta=\tan ^{-1} \frac{y}{x}, \quad r=\sqrt{x^{2}+y^{2}} .
$$


It is interesting to notice that in the case of a source the zero stream line, which forms the walls of the "half body" when $K=0$, is no longer symmetrical with respect to the flow direction. The velocity, and hence the pressure, along

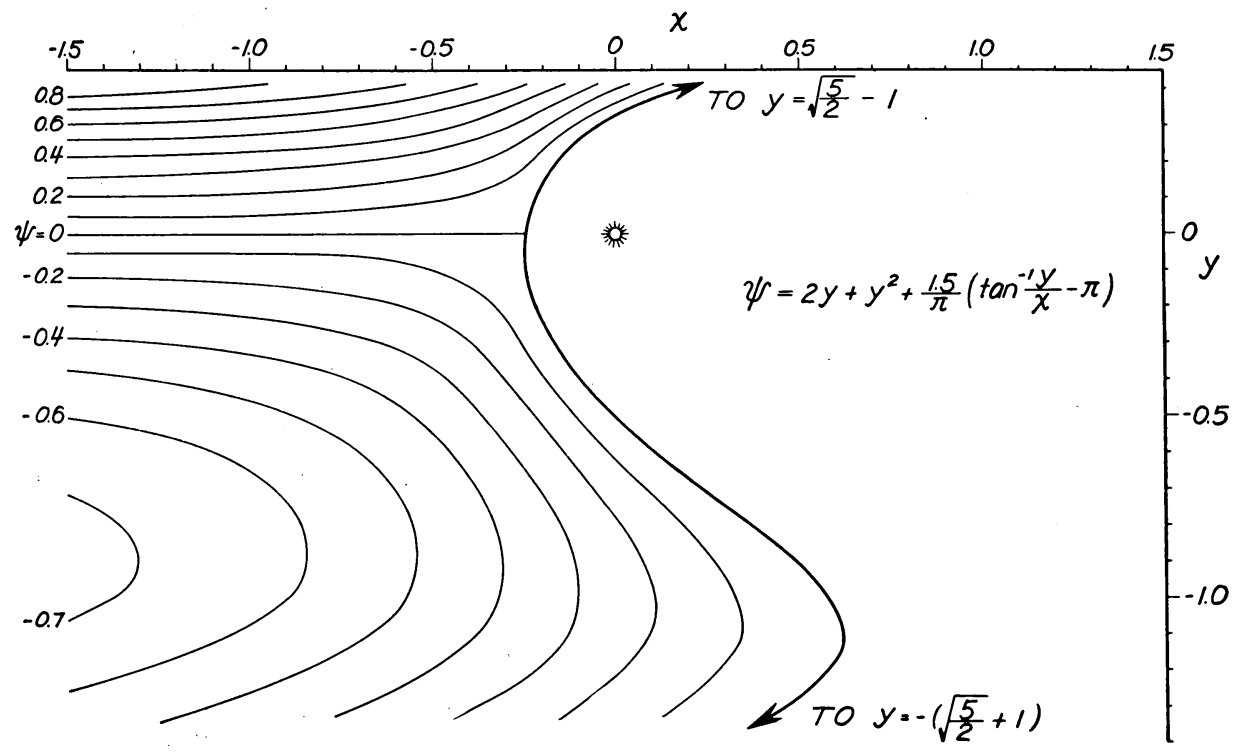

FIG. 3. Source in a Shear Flow.

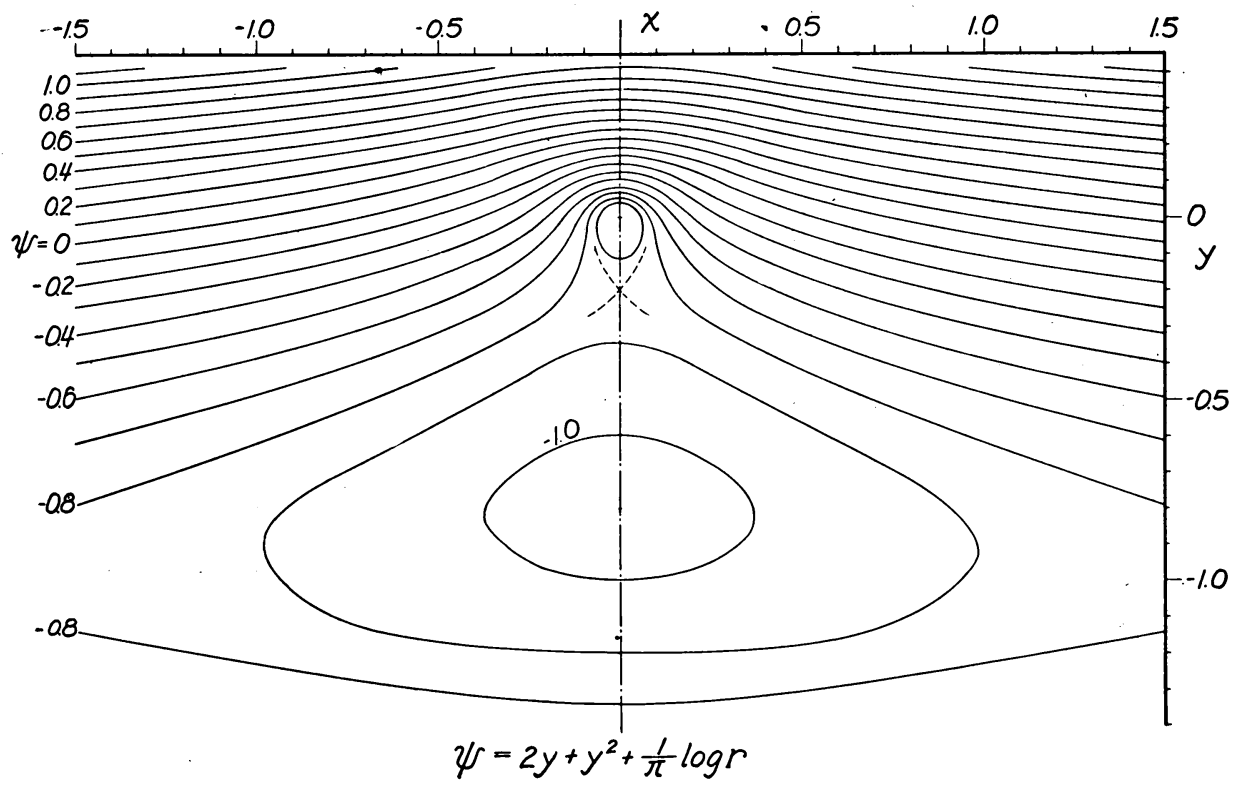

FIG. 4. Vortex in a Shear Flow. 
these two branches are also different. If the flow field within the zero stream line is replaced by a solid body, such lack of balance may cause the resultant pressure force to become infinitely large with the infinitely long solid boundary. This surmise will be verified later when the resultant force and moment are calculated.

If the boundary of the solid body is given, the flow problem indicated in Fig. 1 subjects $\psi_{1}$ to following conditions:

(i) the disturbance velocity due to $\psi_{1}$ must vanish at points far from the body so that Eq. (1) is satisfied;

(ii) the normal component of the disturbance velocity at the surface of the body must equal the negative of that due to $\psi_{0}$ so that the resultant normal velocity is equal to zero.

3. Shear flow over a circular cylinder. To illustrate this method before solving the more complicated case of an airfoil, the flow over a circular cylinder will be investigated first. If the center of the circle is located at the origin, and the radius of the circle is $c / 2$, the undisturbed velocity $U$ due to $\psi_{0}$ at the surface of the cylinder is

$$
U=U_{0}\left(1+\frac{K}{2} \sin \theta\right)
$$

where $\theta$ is given by Eq. (12). The normal component $U_{r}$ of the velocity $U$ is

$$
U_{r}=U \cos \theta=U_{0}\left(\cos \theta+\frac{K}{4} \sin 2 \theta\right) .
$$

Therefore, the normal component of the disturbance velocity due to $\psi_{1}$ at the surface of the circular cylinder must be equal to $-U_{r}$, or

$$
\left(\frac{1}{r} \frac{\partial \psi_{1}}{\partial \theta}\right)_{r=c / 2}=-U_{0}\left(\cos \theta+\frac{K}{4} \sin 2 \theta\right)
$$

where $r$ is given by Eq. (12).

On the other hand, the solution of Eq. (9) that will give vanishing disturbance velocities at points far from the origin is

$$
\psi_{1}=U_{0}\left[a_{0} \log r+b_{0} \theta+\sum_{n=1}^{\infty}\left(a_{n} \cos n \theta+b_{n} \sin n \theta\right) \frac{1}{r^{n}}\right] .
$$

The $a_{n}$ and $b_{n}$ are undetermined coefficients. By substituting Eq. (16) into Eq. (15), one has immediately

$$
\begin{aligned}
& b_{1}=-\left(\frac{c}{2}\right)^{2} \\
& a_{2}=\left(\frac{c}{2}\right)^{3} \frac{K}{8} .
\end{aligned}
$$


All the other coefficients vanish. Therefore, the resultant stream function is $\psi=\psi_{0}+\psi_{1}=U_{0}\left[\left(r-\frac{c^{2}}{4 r}\right) \sin \theta+\frac{K}{2}\left(\frac{r^{2}}{c} \sin ^{2} \theta+\frac{c^{3}}{32 r^{2}} \cos 2 \theta\right)\right]$.

At the surface of the cylinder, $r=c / 2$, Eq. (18) reduces to

$$
\psi=U_{0} \frac{K c}{16}
$$

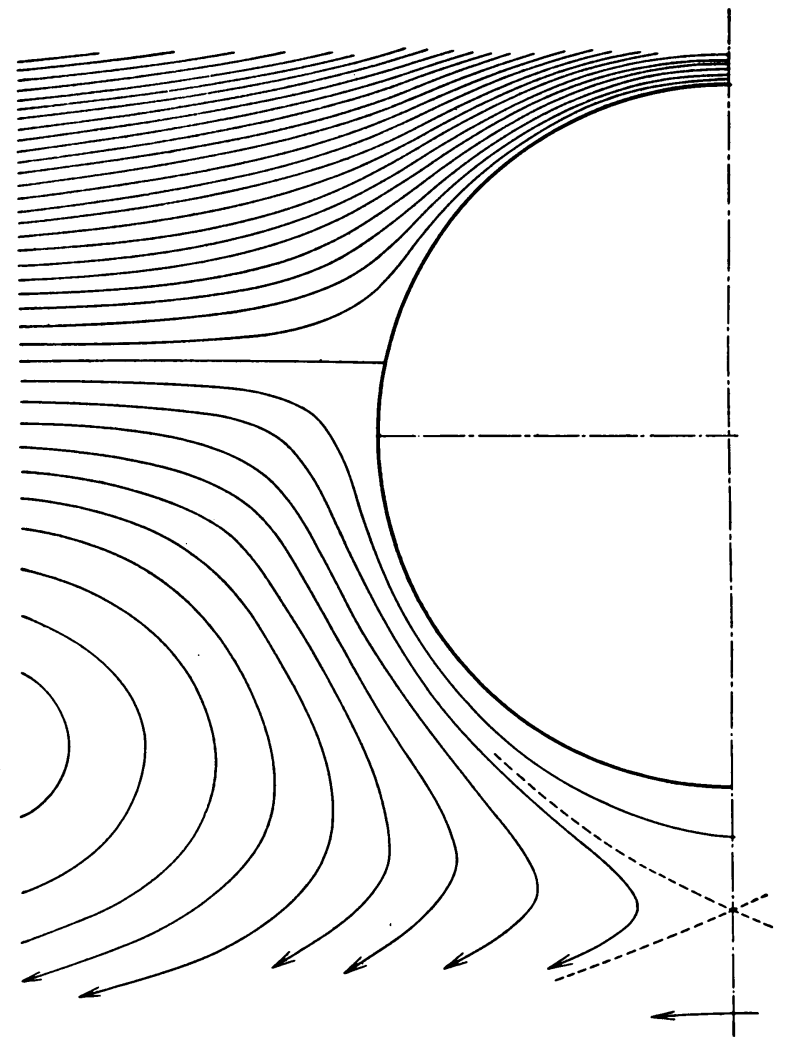

FIG. 5. Circular Cylinder in a Shear Flow with $K=2$.

which is a constant, verifying the boundary condition of the problem. The stagnation point on the cylinder can be calculated by means of Eq. (18). The condition is $(\partial \psi / \partial r)_{r-c / 2}=0$. This is satisfied at the point where

$$
\sin \theta=\frac{1}{K}\left[\sqrt{1+\frac{K^{2}}{4}} \pm 1\right] .
$$

Therefore, if $-8 / 3<K<8 / 3$, there will be two stagnation points on the cyl- 
inder located on that half of the surface where the velocity is higher. If $K$ has a value beyond this range, there will be four stagnation points on the surface.

Fig. 5 shows the stream lines for the case of $K=2$. It is seen that there is an additional stagnation point in the flow field on the negative $y$-axis.

4. Force and moment. In this section, the force and the moment acting on a body whose disturbance stream function $\psi_{1}$ is given by Eq. (16) will be calculated.

The pressure $p$ in the fluid is related to the velocity components $u, v$ and the density $\rho$ by Euler's equations

$$
\begin{aligned}
& \rho u \frac{\partial u}{\partial x}+\rho v \frac{\partial u}{\partial y}=-\frac{\partial p}{\partial x} \\
& \rho u \frac{\partial v}{\partial x}+\rho v \frac{\partial v}{\partial y}=-\frac{\partial p}{\partial y} .
\end{aligned}
$$

By means of Eqs. (4), (7) and (16), the quantities on the left hand side of Eq. (21) can be calculated and then $p$ can be obtained by integration. However in the final calculation of force and moment, the pressure will be integrated along a contour far away from the body; only terms up to $1 / r^{2}$ in $p$ need to be considered. Furthermore, the calculation can be simplified to a certain extent by differentiating the first of Eq. (21) with respect to $x$ and the second with respect to $y$ and adding the resultant. Then by using Eqs. (3) and (5),

$$
\frac{1}{\rho} \nabla^{2} p=2\left[\frac{\partial^{2} \psi_{1}}{\partial x^{2}} \frac{\partial^{2} \psi_{1}}{\partial y^{2}}-\left(\frac{\partial^{2} \psi_{1}}{\partial x \partial y}\right)^{2}+\frac{U_{0} K}{c} \frac{\partial^{2} \psi_{1}}{\partial x^{2}}\right] .
$$

By substituting Eq. (16) into Eq. (22), the differential equation for $p$ is then

$$
\begin{aligned}
\frac{1}{\rho} \nabla^{2} p= & \frac{1}{\rho}\left[\frac{1}{r} \frac{\partial}{\partial r}\left(r \frac{\partial p}{\partial r}\right)+\frac{1}{r^{2}} \frac{\partial^{2} p}{\partial \theta^{2}}\right] \\
= & 2 U_{0}^{2}\left[-\frac{1}{r^{4}}\left(a_{0}^{2}+b_{0}^{2}\right)+\frac{K}{c}\left\{\frac{1}{r^{2}}\left(-a_{0} \cos 2 \theta+b_{0} \sin 2 \theta\right)\right.\right. \\
& +\frac{1}{r^{3}}\left(2 a_{1} \cos 3 \theta+2 b_{1} \sin 3 \theta\right) \\
& \left.\left.+\frac{1}{r^{4}}\left(6 a_{2} \cos 4 \theta+6 b_{2} \sin 4 \theta\right)+\cdots\right\}\right]
\end{aligned}
$$

The appropriate solution for this non-homogeneous equation is evidently 


$$
\begin{aligned}
\frac{1}{\rho} p= & 2 U_{0}^{2}\left[-\frac{1}{4 r^{2}}\left(a_{0}^{2}+b_{0}^{2}\right)+\frac{K}{c}\left\{-\frac{1}{4}\left(-a_{0} \cos 2 \theta+b_{0} \sin 2 \theta\right)\right.\right. \\
& -\frac{1}{8 r}\left(2 a_{1} \cos 3 \theta+2 b_{1} \sin 3 \theta\right) \\
& \left.\left.-\frac{1}{12 r^{2}}\left(6 a_{2} \cos 4 \theta+6 b_{2} \sin 4 \theta\right)\right\}\right] \\
& +A+A_{0} \log r+B_{0} \theta+\frac{1}{r}\left(A_{1} \cos \theta+B_{1} \sin \theta\right) \\
& +\frac{1}{r^{2}}\left(A_{2} \cos 2 \theta+B_{2} \sin 2 \theta\right)+\cdots
\end{aligned}
$$

where the $A$ 's and $B$ 's are undetermined constants. Either the first or the second equation (21) can be used to determine $A_{0}, A_{1}, \cdots$ and $B_{0}, B_{1}, B_{2}, \cdots$. The final result for the pressure $p$ can be written as

$$
\begin{aligned}
\frac{p}{\rho}=A & +U_{0}^{2}\left[\frac{K}{c} a_{0} \log r+\frac{K}{c} b_{0} \theta-\frac{K}{2 c}\left(-a_{0} \cos 2 \theta+b_{0} \sin 2 \theta\right)\right. \\
& -\frac{K}{2 c r}\left(a_{1} \cos 3 \theta+b_{1} \sin 3 \theta\right) \\
& +\frac{1}{r}\left\{\left(\frac{3}{2} \frac{K a_{1}}{c}-b_{0}\right) \cos \theta+\left(\frac{3}{2} \frac{K b_{1}}{c}-a_{0}\right) \sin \theta\right\} \\
& +\frac{1}{r^{2}}\left\{-\frac{a_{0}^{2}+b_{0}^{2}}{2}-\frac{K}{c}\left(a_{2} \cos 4 \theta+b_{2} \sin 4 \theta\right)\right. \\
& \left.\left.+\left(2 \frac{K a_{2}}{c}-b_{1}\right) \cos 2 \theta+2\left(\frac{K b_{2}}{c}+a_{1}\right) \sin 2 \theta\right\}+\cdots\right]
\end{aligned}
$$

Now by considering the pressure force and momentum of the fluid, the following relations can be obtained between the forces $X, Y$ acting on the body and their moment $M_{0}$ about the origin' (Fig. 1).

$$
\begin{aligned}
X & =-\int_{C} p d y-\int_{C} \rho u(u d y-v d x) \\
Y & =\int_{C} p d x-\int_{C} \rho v(u d y-v d x) \\
M_{0} & =\int_{C} p(x d x+y d y)-\int_{C} \rho(v x-u y)(u d y-v d x) .
\end{aligned}
$$

${ }^{2}$ See for example Glauert, H., Aerofoil and airscrew theory, Cambridge University Press, London, 1930, p. 80. 
The integrals are taken along any closed curve $C$ enclosing the body. If a circle with radius $R$ is taken as the contour, then in the integrals of Eq. (26)

$$
\begin{aligned}
& x=R \cos \theta \\
& y=R \sin \theta .
\end{aligned}
$$

Therefore, by using Eq. (25),

$$
-\int_{C} p d y=-R \int_{0}^{2 \pi} p \cos \theta d \theta=\pi \rho U_{0}^{2}\left[b_{0}-\frac{3}{2} \frac{K}{c} a_{1}\right] .
$$

Furthermore,

$$
\begin{aligned}
u d y-v d x= & U_{0}\left[\frac{K}{2 c} R^{2} \sin 2 \theta+R \cos \theta+b_{0}+\frac{1}{R}\left(-a_{1} \sin \theta+b_{1} \cos \theta\right)\right. \\
& \left.+\frac{1}{R^{2}}\left(-2 a_{2} \sin 2 \theta+2 b_{2} \cos 2 \theta\right)+\cdots\right] d \theta
\end{aligned}
$$

and

$$
\begin{aligned}
u= & U_{0}\left[\frac{K}{c} R \sin \theta+1+\frac{1}{R}\left(a_{0} \sin \theta+b_{0} \cos \theta\right)\right. \\
& \left.+\frac{1}{R^{2}}\left(-a_{1} \sin 2 \theta+b_{1} \cos 2 \theta\right)+\cdots\right] .
\end{aligned}
$$

By combining Eqs. (29) and (30), the second term in the equation for the horizontal force $X$ can be calculated as

$$
-\int_{C} \rho u(u d y-v d x)=-\pi \rho U_{0}^{2}\left[3 b_{0}-\frac{3 K}{2 c} a_{1}\right] .
$$

Finally the horizontal force is expressed as

$$
X=-2 \pi \rho U_{0}^{2} b_{0} \text {. }
$$

Similarly, the vertical force $Y$ or lift and the moment $M_{0}$ about the origin are obtained in the following forms:

$$
\begin{aligned}
Y & =2 \pi \rho U_{0}^{2}\left[a_{0}+\frac{K}{c}\left(R b_{0}-b_{1}\right)\right] . \\
M_{0} & =2 \pi \rho U_{0}^{2}\left[a_{0} b_{0}-a_{1}+\frac{K}{c}\left(\frac{1}{2} R^{2} b_{0}-b_{2}\right)\right] .
\end{aligned}
$$

Eqs. (32), (33) and (34) show that the force and moment on the body can be calculated in terms of the strength of the vortex, the source, the doublets 
and the quadruplets in the disturbance stream function $\psi_{1}$. These equations can be regarded as the generalization of the well-known Blasius formulas. They reduce to the latter formulas if $K=0$. However, it should be noticed that if there is a source, i.e., $b_{0} \neq 0$, both the lift $Y$ and the moment $M_{0}$ grow to infinite magnitude with $R \rightarrow \infty$. This confirms the surmise stated previously. If the boundary of the body is closed, $b_{0}$ must vanish, and there can be no horizontal force or drag and the lift and moment will remain finite. v. Sanden ${ }^{1}$ obtained a small drag force for his wedge shaped body. Evidently this is due to the unavoidable inaccuracies in his numerical method.

5. Symmetrical Joukowsky airfoils. For the flow over a symmetrical Joukowsky airfoil, it is difficult to determine the disturbance stream function $\psi_{1}$ directly. But, as seen from Eq. (9), $\psi_{1}$ satisfies Laplace's equation, and therefore conformal transformation can be used. It should be noticed, however, that $\psi_{0}$ does not satisfy Laplace's equation; and therefore it does not allow the use of this transformation in the ordinary sense.

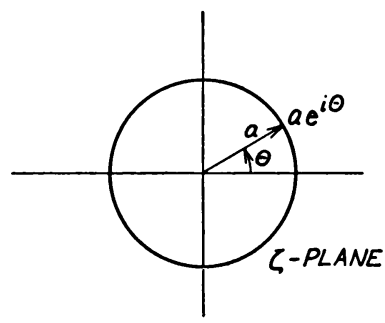

(a)

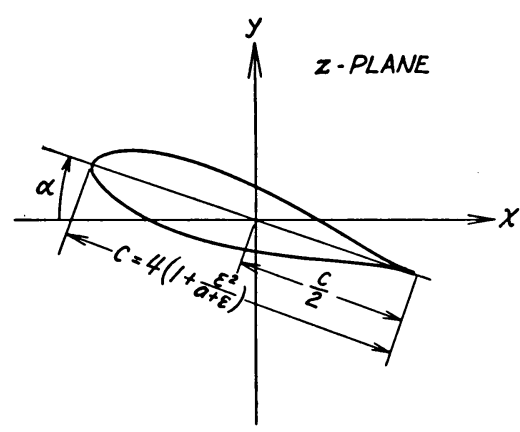

(b)

FIG. 6. Joukowsky Transformation of a Circle into an Airfoil.

Consider a circle of radius $a$ in the $\zeta$ plane (Fig. 6a). The transformation

$$
z=e^{i \alpha}\left(\zeta-\epsilon+\frac{1}{\zeta-\epsilon}+\frac{2 \epsilon^{2}}{a+\epsilon}\right)
$$

will transform the circle into a symmetrical Joukowsky airfoil in the $z$ plane 
(Fig. 6b) inclined at an angle $\alpha$ against the $x$-axis, $\dot{\epsilon}$ is a positive quantity which increases with the thickness of the airfoil. $\epsilon$ is zero for a flat plate and is infinite for a circle. Furthermore

$$
a=1+\epsilon .
$$

The origin of the $z$-plane lies at the center of the airfoil (Fig. 6b). If we represent the circle in the $\zeta$-plane by

$$
\zeta=a e^{i \theta},
$$

the trailing edge of the airfoil corresponds to the point where $\theta=0$. The leading edge of the airfoil corresponds to the point where $\theta=\pi$. Therefore, the chord is

With

$$
c=4\left(1+\frac{\epsilon^{2}}{a+\epsilon}\right) \text {. }
$$

$$
\epsilon / a=\lambda
$$

the values of $x$ and $y$ corresponding to an arbitrary value of $\theta$ are given by Eq. (35) as

$$
\begin{aligned}
& x=a\left\{\cos (\theta-\alpha)-\lambda \cos \alpha+\frac{\cos (\theta+\alpha)-\lambda \cos \alpha}{a^{2}\left(1-2 \lambda \cos \theta+\lambda^{2}\right)}+\frac{2 \lambda^{2} \cos \alpha}{1+\lambda}\right\} \\
& y=a\left\{\sin (\theta-\alpha)+\lambda \sin \alpha-\frac{\sin (\theta+\alpha)-\lambda \sin \theta}{a^{2}\left(1-2 \lambda \cos \theta+\lambda^{2}\right)}-\frac{2 \lambda^{2} \sin \alpha}{1+\lambda}\right\} .
\end{aligned}
$$

After Eq. (7), the velocity due to $\psi_{0}$ at a point in the $z$-plane corresponding to $\zeta=a e^{i \theta}$ is

$$
U=U_{0}\left[1+\frac{K a}{c}\left\{\sin (\theta-\alpha)-\frac{\sin (\theta+\alpha)-\lambda \sin \alpha}{a^{2}\left(1-2 \lambda \cos \theta+\lambda^{2}\right)}+\frac{\lambda \sin \alpha}{a(1+\lambda)}\right\}\right]
$$

This velocity is horizontal and has a tangential component in the counterclockwise direction equal to

$$
-U\left[\frac{d x}{\sqrt{(d x)^{2}+(d y)^{2}}}\right]_{\zeta=a e^{i \theta}}
$$

and a normal component, directed along the outward normal, equal to

$$
U\left[\frac{d y}{\sqrt{(d x)^{2}+(d y)^{2}}}\right]_{\zeta=a e^{i \theta}} \cdot
$$

On the other hand if $\psi_{1}$ is the disturbance stream function, the velocity component normal to the circle $\zeta=a e^{i \theta}$ is 


$$
\left(\frac{1}{r} \frac{\partial \psi_{1}}{\partial \theta}\right)_{r=a}
$$

Therefore, the corresponding velocity component in the $z$-plane, normal to the surface of the airfoil, is

$$
\left(\frac{1}{r} \frac{\partial \psi_{1}}{\partial \theta}\right)_{r=a}\left|\frac{d \zeta}{d z}\right|_{\zeta=a e^{i \theta}}
$$

Then the boundary condition at the airfoil surface requires that this normal component of the disturbance velocity be equal to the negative of the normal velocity component due to $\psi_{0}$. By means of Eqs. (42) and (44), this boundary condition is expressed by

But

$$
\left(\frac{1}{r} \frac{\partial \psi_{1}}{\partial \theta}\right)_{r=a}\left|\frac{d \zeta}{d z}\right|_{\zeta=a e^{i \theta}}=-U\left[\frac{d y}{\sqrt{(d x)^{2}+(d y)^{2}}}\right]_{\zeta=a e^{i \theta}} .
$$

$$
\left[\sqrt{(d x)^{2}+(d y)^{2}}\right]_{\zeta=a e^{i \theta}}=\frac{a d \theta}{\left|\frac{d \zeta}{d z}\right|_{\zeta=a e^{i \theta}}} \cdot
$$

With the aid of Eqs. (39), (40), and (42), Eq. (45) can be re-written as follows

$$
\begin{aligned}
\frac{1}{U_{0}}\left(\frac{1}{r} \frac{\partial \psi_{1}}{\partial \theta}\right)_{r=a} & \\
=- & {\left[1+\frac{K a}{c}\left\{\sin (\theta-\alpha)-\frac{\sin (\theta+\alpha)-\lambda \cos \alpha}{a^{2}\left(1-2 \lambda \cos \theta+\lambda^{2}\right)}+\frac{\lambda \sin \alpha}{a(1+\lambda)}\right\}\right] } \\
& {\left[\cos (\theta-\alpha)-\frac{\cos (\theta+\alpha)+\lambda \cos (\theta-\alpha)+2 \lambda \cos \alpha}{a^{2}\left(1-2 \lambda \cos \theta+\lambda^{2}\right)^{2}}\right] . }
\end{aligned}
$$

This equation alone would not determine the function $\psi_{1}$ completely but for the additional so-called Kutta-Joukowsky condition, which fixes the strength of the circulation over the airfoil.

6. Strength of circulation. The Kutta-Joukowsky condition states that the velocity at the trailing edge of the airfoil must be finite. The velocity at the trailing edge of the airfoil consists of two parts: one part is that due to $\psi_{0}$ and the other that due to $\psi_{1}$. Only tangential components need be considered because the normal components cancel each other as required by the boundary condition. The part due to $\psi_{1}$ in the counterclockwise direction is

$$
-\frac{\partial \psi_{1}}{\partial r}\left|\frac{d \zeta}{d z}\right|_{\zeta=a}
$$

Therefore, by means of Eq. (41), the resultant velocity at the trailing edge is 


$$
\left[-\frac{\partial \psi_{1}}{\partial r}-U \frac{d x}{\sqrt{(d x)^{2}+(d y)^{2}}} \frac{1}{\left|\frac{d \zeta}{d z}\right|_{\zeta=a}}\right]\left|\frac{d \zeta}{d z}\right|_{\zeta=a}
$$

but $\zeta=a$ is the singular point of the transformation from $\zeta$ to $z$ as can be easily verified. In other words, $|d \zeta / d z|$ tends to infinity at $\zeta=a$. Therefore, the resultant velocity at the trailing edge can only be finite if the quantity within the square bracket of Eq. (49) vanishes. By means of Eqs. (39), (40), (46), this condition can be written as

$$
\begin{gathered}
-\left(\frac{\partial \psi_{1}}{\partial r}\right)_{\substack{r=a \\
\theta=0}}-U_{0}\left[1+\frac{K a}{c}\left\{\sin (\theta-\alpha)-\frac{\sin (\theta+\alpha)-\lambda \cos \alpha}{a^{2}\left(1-2 \lambda \cos \theta+\lambda^{2}\right)}+\frac{\lambda \sin \alpha}{a(1+\lambda)}\right\}\right] \\
\cdot\left[\sin (\theta-\alpha)+\frac{\sin (\theta+\alpha)-\lambda^{2} \sin (\theta-\alpha)-2 \lambda \sin \alpha}{a^{2}\left(1-2 \lambda \cos \theta+\lambda^{2}\right)}\right]_{\theta=0}=0 .
\end{gathered}
$$

The appropriate general solution $\psi_{1}$ in the $\zeta$-plane is

Therefore,

$$
\psi_{1}=U_{0}\left[\alpha_{0} \log r+\beta_{0} \theta+\sum_{n=1}^{\infty}\left(\alpha_{n} \cos n \theta+\beta_{n} \sin n \theta\right) \frac{1}{r^{n}}\right]
$$

$$
\begin{aligned}
& \frac{1}{U_{0}}\left(\frac{1}{r} \frac{\partial \psi_{1}}{\partial \theta}\right)_{r=a}=\frac{\beta_{0}}{a}+\sum_{n=1}^{\infty}\left(-n \alpha_{n} \sin n \theta+n \beta_{n} \cos n \theta\right) \frac{1}{a^{n+1}}, \\
& -\frac{1}{U_{0}}\left(\frac{\partial \psi_{1}}{\partial r}\right)_{r=a}=-\frac{\alpha_{0}}{a}+\sum_{n=1}^{\infty}\left(n \alpha_{n} \cos n \theta+n \beta_{n} \sin n \theta\right) \frac{1}{a^{n+1}} .
\end{aligned}
$$

By expanding the right hand side of Eq. (47) into a trigonometric series, all the coefficients $\alpha_{1}, \alpha_{2}, \cdots$ and $\beta_{0}, \beta_{1}, \cdots$ in $\psi_{1}$ can be determined by the first of Eqs. (52). Then the second equation of Eqs. (52) together with Eq. (50) will determine the value of $\alpha_{0}$. Actually, calculations are easier if each term on the right of Eq. (47) is taken separately. For example, the term $-U_{0} \cos (\theta-\alpha)$ can be expanded into $-U_{0} \cos \alpha \cos \theta-U_{0} \sin \alpha \sin \theta$. Then according to Eq. (52), the contribution to $\alpha_{1}$ is $a^{2} \sin \alpha$; the contribution to $\beta_{1}$ is $-a^{2} \cos \alpha$. Finally the contribution to $-\left(\partial \psi_{1} / \partial r\right)_{r=a}$ is $U_{0}(\sin \alpha \cos \theta$ $-\cos \alpha \sin \theta)=-U_{0} \sin (\theta-\alpha)$. The other more complicated terms can be treated in a similar manner with the aid of the following expansions and their derivatives with respect to $\theta$ and $\lambda$ :

$$
\begin{aligned}
& \frac{\sin \theta}{1-2 \lambda \cos \theta+\lambda^{2}}=\sum_{n=1}^{\infty} \lambda^{n-1} \sin n \theta \\
& \frac{\cos \theta-\lambda}{1-2 \lambda \cos \theta+\lambda^{2}}=\sum_{n=1}^{\infty} \lambda^{n-1} \cos n \theta \\
& \frac{1}{1-2 \lambda \cos \theta+\lambda^{2}}=\frac{1}{1-\lambda^{2}}\left(1+2 \sum_{n=1}^{\infty} \lambda^{n} \cos n \theta\right) .
\end{aligned}
$$


The value of $-\left(\partial \psi_{1} / \partial r\right)_{r=a}$ thus found is

$$
\begin{gathered}
-\frac{1}{U_{0}}\left(\frac{\partial \psi_{1}}{\partial r}\right)_{r=a}=-\frac{\alpha_{0}}{a}-\left[1+\frac{K \lambda}{c(1+\lambda)}\right] \\
\cdot\left[\sin (\theta-\alpha)-\frac{\sin (\theta+\alpha)-\lambda^{2} \sin (\theta-\alpha)-2 \lambda \sin \alpha}{a^{2}\left(1-2 \lambda \cos \theta+\lambda^{2}\right)}\right] \\
+\frac{K a}{c}\left[\frac{1}{2} \cos 2(\theta-\alpha)+\left\{\sin (\theta-\alpha)-\frac{\sin (\theta+\alpha)-\lambda \cos \alpha}{a^{2}\left(1-2 \lambda \cos \theta+\lambda^{2}\right)}\right\}\right. \\
\quad \frac{\sin (\theta+\alpha)-\lambda^{2} \sin (\theta-\alpha)-2 \lambda \sin \alpha}{a^{2}\left(1-2 \lambda \cos \theta+\lambda^{2}\right)^{2}} \\
\left.\quad-\frac{\cos (\theta-\alpha)[\cos (\theta+\alpha)-\lambda \cos \alpha\rfloor}{a^{2}\left(1-2 \lambda \cos \theta+\lambda^{2}\right)}+\frac{1}{2 a^{4}\left(1-\lambda^{2}\right)\left(1-2 \lambda \cos \theta+\lambda^{2}\right)}\right] .
\end{gathered}
$$

The strength of circulation $\alpha_{0}$ necessary to satisfy the Kutta-Joukowsky condition can then be calculated by means of Eq. (50). The result is

$$
\alpha_{0}=a U_{0}\left[2 \sin \alpha+\frac{K a}{c}\left\{\frac{2 \lambda \sin ^{2} \alpha}{a(1+\lambda)}-\frac{1}{2}+\cos 2 \alpha-\frac{1}{a}+\frac{1}{2 a(1+\lambda)}\right\}\right]
$$

The strength of the source, $\beta_{0}$, is zero, as would be expected from the fact that the airfoil is represented by a closed contour.

7. Strengths of doublets and quadruplets. By collecting the contributions of the different terms of $\left[(1 / r) \partial \psi_{1} / \partial \theta\right]_{r=a}$ in Eq. (47) to $\alpha_{1}, \alpha_{2}, \beta_{1}$ and $\beta_{2}$, the following values of the strength of doublets and quadruplets in the $\zeta$-plane are obtained

$$
\begin{aligned}
\alpha_{1}= & a^{2} U_{0}\left[\left(1+\frac{1}{a^{2}}\right) \sin \alpha\right. \\
& \left.+\frac{K a}{c}\left\{\frac{\lambda}{2 a^{2}} \cos 2 \alpha-\frac{\lambda}{2 a^{4}\left(1-\lambda^{2}\right)}+\left(1+\frac{1}{a^{2}}\right) \frac{\lambda \sin ^{2} \alpha}{a(1+\lambda)}\right\}\right] \\
\beta_{1}= & a^{2} U_{0}\left[\left(-1+\frac{1}{a^{2}}\right) \cos \alpha+\frac{K a}{c} \sin 2 \alpha\left\{-\frac{\lambda}{2 a^{2}}+\left(-1+\frac{1}{a^{2}}\right) \frac{\lambda}{2 a(1+\lambda)}\right\}\right] \\
\alpha_{2}= & a^{3} U_{0}\left[\frac{\lambda}{a^{2}} \sin \alpha+\frac{K a}{c} \cos 2 \alpha\left\{\frac{1}{4}-\frac{1}{2 a^{2}}+\frac{\lambda^{2}}{2 a^{2}}+\frac{1-3 \lambda^{2}}{4 a^{4}\left(1-\lambda^{2}\right)}\right\}\right] \\
\beta_{2}= & a^{3} U_{0}\left[\frac{\lambda}{a^{2}} \cos \alpha+\frac{K a}{c} \sin 2 \alpha\left\{\frac{1}{4}-\frac{\lambda^{2}}{2 a^{2}}-\frac{1-3 \lambda^{2}}{4 a^{4}\left(1-\lambda^{2}\right)}\right\}\right] .
\end{aligned}
$$

However, to calculate the lift and moment over the airfoil, it is not the strength of doublets and quadruplets in the $\zeta$-plane that is needed but the strength of those in the $z$-plane, where the airfoil is located. From the known 
values of $\alpha_{1}, \cdots \beta_{2}$ given by Eq. (35) and the transformation specified by Eq. (55), the desired quantities can be easily calculated in the following manner.

By introducing the conjugate function $\phi_{1}$ of the stream function one can write

$$
\phi_{1}+i \psi_{1}=i \alpha_{0} \log \zeta+\frac{-\beta_{1}+i \alpha_{1}}{\zeta}+\frac{-\beta_{2}+i \alpha_{2}}{\zeta^{2}}+\cdots .
$$

With $\zeta=r e^{i \theta}$, the real part of Eq. (56) is Eq. (51) since $\beta_{0}$ is found to be zero. Now Eq. (35) gives

$$
\zeta=z e^{i \alpha}\left[1+\frac{\lambda}{(1+\lambda) z e^{i \alpha}}-\frac{1}{z^{2} e^{2 i \alpha}}+\cdots\right]
$$

for sufficiently large values of $z$. Then $\phi_{1}+i \psi_{1}$ can be expanded into a series in $z$ by substituting Eq. (57) into Eq. (56). The result is

$$
\begin{aligned}
\phi_{1}+i \psi_{1} & =i \alpha_{0} \log z+\left[-\beta_{1}+i\left(\alpha_{1}+\frac{\lambda \alpha_{0}}{1+\lambda}\right)\right] z^{-1} e^{-i \alpha} \\
& +\left[-\beta_{2}+\frac{\lambda \beta_{1}}{1+\lambda}+i\left(\alpha_{2}-\frac{\lambda \alpha_{1}}{1+\lambda}-\alpha_{0}-\frac{\lambda^{2} \alpha_{0}}{2(1+\lambda)^{2}}\right)\right] z^{-2} e^{-2 i \alpha}+\cdots
\end{aligned}
$$

If the strength of the circulation, the doublets, and the quadruplets in the $z$-plane are denoted by $a_{0}, a_{1}, b_{1}, a_{2}$ and $b_{2}$, respectively, the following relations are obtained by comparing Eq. (58) with Eq. (56):

$$
\begin{aligned}
& a_{0}=\alpha_{0}, \quad b_{0}=0 \\
& a_{1}=\beta_{1} \sin \alpha+\left(\alpha_{1}+\frac{\lambda \alpha_{0}}{1+\lambda}\right) \cos \alpha \\
& b_{1}=\beta_{1} \cos \alpha-\left(\alpha_{1}+\frac{\lambda \alpha_{0}}{1+\lambda}\right) \sin \alpha \\
& a_{2}=\left(\beta_{2}-\frac{\lambda \beta_{1}}{1+\lambda}\right) \sin 2 \alpha+\left(\alpha_{2}-\frac{\lambda \alpha_{1}}{1+\lambda}-\alpha_{0}-\frac{\lambda^{2} \alpha_{0}}{2(1+\lambda)^{2}}\right) \cos 2 \alpha \\
& b_{2}=\left(\beta_{2}-\frac{\lambda \beta_{1}}{1+\lambda}\right) \cos 2 \alpha-\left(\alpha_{2}-\frac{\lambda \alpha_{1}}{1+\lambda}-\alpha_{0}-\frac{\lambda^{2} \alpha_{0}}{2(1+\lambda)^{2}}\right) \sin 2 \alpha .
\end{aligned}
$$

Eqs. (54), (55), and (59) give all the necessary data to calculate the forces and moment acting on the airfoil.

8. Lift and moment coefficients. By means of Eqs. (32), (33), and (34), the drag, lift, and moment about the origin can be calculated with the values of $a_{0}, a_{1}, a_{2}, b_{0}, b_{1}, b_{2}$ given by Eq. (59). It is seen that the drag is also zero in 
the case of shear flow. The result of the computation can be conveniently represented by defining the functions $l_{0}, l_{1}, l_{2}, l_{3}, l_{4}$ and $m_{0}, m_{1}, m_{2}, m_{3}, m_{4}$ in connection with the lift coefficient $C_{L}$ and moment coefficient $C_{M_{0}}$ in the following manner:

$$
\begin{aligned}
& C_{L}=\frac{Y}{\frac{1}{2} \rho U_{0}^{2} c}=2 \pi\left[l_{0} \sin \alpha+K\left\{l_{1}+l_{2} \cos 2 \alpha\right\}+K^{2}\left\{l_{3} \sin \alpha+l_{4} \sin 3 \alpha\right\}\right] \\
& C_{M_{0}}=\frac{-M_{0}}{\frac{1}{2} \rho U_{0}^{2} c^{2}}=\frac{\pi}{2}\left[\frac{m_{0}}{2} \sin 2 \alpha+K\left\{m_{1} \cos \alpha+m_{2} \cos 3 \alpha\right\}\right. \\
& \left.+K^{2}\left\{m_{3} \sin \alpha+m_{4} \sin 4 \alpha\right\}\right] \text {. }
\end{aligned}
$$

The negative sign in the moment coefficient is introduced in accordance with the usual convention of taking the stalling moment as positive. The functions $l_{0}, l_{1}, l_{2}, l_{3}, l_{4}$ and $m_{0}, m_{1}, m_{2}, m_{3}, m_{4}$ are given by the following equations:

$$
\begin{aligned}
& l_{0}=\frac{1}{h}, \quad h=\frac{1}{a}+\frac{\lambda^{2}}{1+\lambda} \\
& l_{1}=\frac{\lambda(\lambda+2 / a)}{8 h^{2}(1+\lambda)}, \quad l_{2}=\frac{\lambda^{2}(2+1 / a)}{8 h^{2}(1+\lambda)} \\
& l_{3}=-\frac{\lambda}{64 h^{3} a(1+\lambda)}\left[\frac{1}{a^{2}(1+\lambda)}-2 \lambda+\lambda^{2}\right] \\
& l_{4}=\frac{\lambda}{64 a^{2} h^{3}}\left[1-\frac{\lambda}{(1+\lambda)^{2}}\right] \\
& m_{0}=\frac{1}{a h^{2}}\left[\frac{1}{a}+\frac{\lambda}{1+\lambda}\right] \\
& m_{1}=\frac{1}{8 a h^{3}}\left[\frac{1}{a}+\frac{\lambda}{2(1+\lambda)}\left(1+\lambda+\frac{\lambda}{a(1+\lambda)}\right)\right] \\
& m_{2}=-\frac{1}{8 a h^{3}}\left[\frac{1}{a}\left(1-\frac{3}{2} \lambda\right)+\frac{\lambda}{2(1+\lambda)}\left(\frac{2}{a^{2}}-\lambda^{2} \frac{2+1 / a}{1+\lambda}\right)\right] \\
& m_{3}=-\frac{1}{64 a h^{4}}\left[\frac{1}{1+\lambda}\left(\frac{1}{a^{2}}+\frac{\lambda^{2}}{2 a^{2}(1+\lambda)^{2}}\right)-\frac{\lambda^{2}}{2 a(1+\lambda)^{2}}\right] \\
& m_{4}=\frac{1}{64 a h^{4}}\left[1+\lambda-\frac{\lambda}{a^{2}(1+\lambda)}\left(1+\frac{\lambda}{a(1+\lambda)}\right)+\frac{\lambda^{2}\left(1+\lambda^{2}\right)}{2 a(1+\lambda)\left(1-\lambda^{2}\right)}\right. \\
& \left.-\frac{1}{4 a^{2}}\left(\frac{1}{a}+\frac{1}{1+\lambda}\right)+\frac{9 \lambda^{2}}{4 a^{2}(1+\lambda)}\right] \text {. }
\end{aligned}
$$


Table I gives the numerical values of these functions together with the thickness ratio $\delta$ of the airfoil. It is seen that with increasing thickness the

TABLE I

\begin{tabular}{l|c|c|c|c|c|c|c|c|c|c|c}
\hline \hline$\epsilon$ & $\delta$ & $l_{0}$ & $l_{1}$ & \multicolumn{1}{|c|}{$l_{2}$} & $l_{3}$ & $l_{4}$ & $m_{0}$ & $m_{1}$ & $m_{2}$ & $m_{2}$ & $m_{4}$ \\
\hline 0 & 0 & 1.0000 & 0 & 0 & 0 & 0 & 1.0000 & 0.1250 & -0.1250 & -0.0156 & 0.0078 \\
0.05 & 0.0618 & 1.0476 & 0.012 & 0.0009 & -0.0006 & 0.0007 & 1.0430 & 0.1334 & -0.1267 & -0.0155 & 0.0102 \\
0.10 & 0.1179 & 1.0909 & 0.0237 & 0.0033 & -0.0009 & 0.0014 & 1.0737 & 0.1413 & -0.1259 & -0.0152 & 0.0128 \\
0.15 & 0.1687 & 1.1304 & 0.0345 & 0.0069 & -0.0010 & 0.0020 & 1.0945 & 0.1477 & -0.1231 & -0.0148 & 0.0157 \\
0.20 & 0.2150 & 1.1667 & 0.0446 & 0.0115 & -0.0009 & 0.0025 & 1.1073 & 0.1530 & -0.1190 & -0.0143 & 0.0188 \\
0.30 & 0.2958 & 1.2308 & 0.0628 & 0.0227 & -0.0003 & 0.0034 & 1.1148 & 0.1610 & -0.1080 & -0.0131 & 0.0250 \\
0.40 & 0.3636 & 1.2857 & 0.0787 & 0.0356 & +0.0005 & 0.0040 & 1.1058 & 0.1660 & -0.0953 & -0.0119 & 0.0310 \\
0.50 & 0.4210 & 1.3333 & 0.0926 & 0.0494 & +0.0014 & 0.0045 & 1.0864 & 0.1687 & -0.0823 & -0.0106 & 0.0365 \\
0.60 & 0.4701 & 1.3750 & 0.1047 & 0.0635 & +0.0023 & 0.0048 & 1.0608 & 0.1697 & -0.0697 & -0.0095 & 0.0416 \\
0.80 & 0.5489 & 1.4444 & 0.1248 & 0.0911 & +0.0039 & 0.0051 & 1.0006 & 0.1683 & -0.0474 & -0.0075 & 0.0499 \\
1.00 & 0.6089 & 1.5000 & 0.1406 & 0.1172 & +0.0051 & 0.0051 & 0.9375 & 0.1641 & -0.0293 & -0.0059 & 0.0560 \\
$\infty$ & 1.0000 & 2.0000 & 0.2500 & 0.5000 & 0 & 0 & 0 & 0 & 0 & 0 & 0 \\
\hline
\end{tabular}

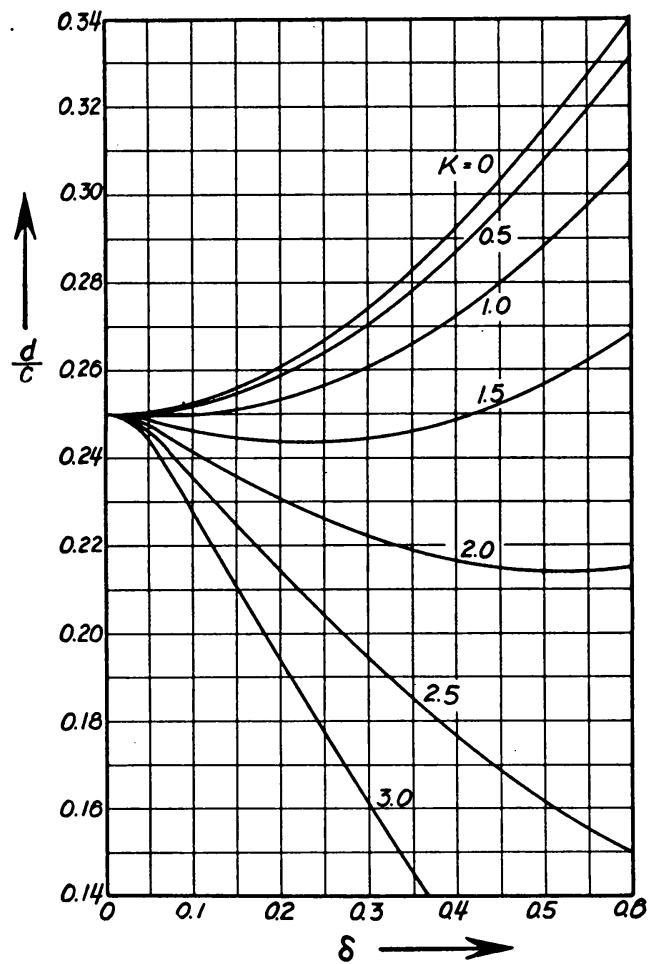

FIG. 7. Ratio of the distance $d$ from leading edge to aerodynamic center (positive when it is behind the leading edge) to the chord $c$ as a function of thickness ratio $\delta$ and non-dimensional velocity gradient $K$. 
effect of the velocity gradient $K$ becomes larger and larger. For example, if $\delta=11.79 \%$ the effect of $K$ on $C_{L}$ at small angles of attack is approximately given by the term $2 \pi K\left(l_{1}+l_{2}\right)=2 \pi K \times 0.02695$. In other words, it is equivalent to a shift in the angle of zero lift by $1.54 K$ degrees. For an airfoil of $21.50 \%$ thickness, this value is $3.21 K$ degrees.

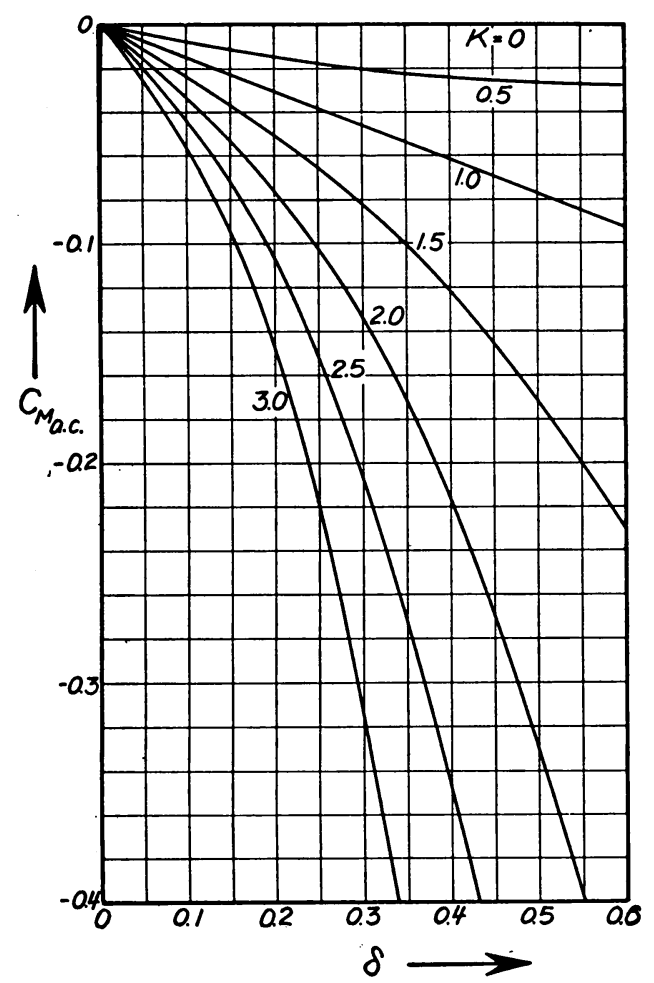

FIG. 8. Moment coefficient $C_{M_{\mathrm{a} .0}}$ about the aerodynamic center (positive for stalling moment) as a function of thickness ratio and non-dimensional velocity gradient $K$.

9. Aerodynamic center. To demonstrate the effect of the velocity gradien $t$ on the moment more clearly, the aerodynamic center for small angles of attack will be calculated presently. For small values of $\alpha$ the expressions for $C_{L}$ and $C_{M_{0}}$ of Eq. (60) can be simplified to

$$
\begin{aligned}
C_{L} & =2 \pi\left[\left\{l_{0}+K^{2}\left(l_{3}+3 l_{4}\right)\right\} \alpha+K\left(l_{1}+l_{2}\right)\right] \\
C_{M_{0}} & =\frac{\pi}{2}\left[\left\{m_{0}+K^{2}\left(2 m_{3}+4 m_{4}\right)\right\} \alpha+K\left(m_{1}+m_{2}\right)\right] .
\end{aligned}
$$

The moment coefficient corresponds to the stalling moment about the center of the airfoil. If the moment is referred to a point on the chord at a 
distance $d$ back of the leading edge, the corresponding moment coefficient $C_{M}$ will be

$$
\begin{aligned}
C_{M}= & C_{M_{0}}-C_{L}\left(\frac{1}{2}-\frac{d}{c}\right) \\
= & \frac{\pi}{2}\left[\left\{m_{0}+K^{2}\left(2 m_{3}+4 m_{4}\right)\right\} \alpha+K\left(m_{1}+m_{2}\right)\right] \\
& -\left(\frac{1}{2}-\frac{d}{c}\right) \cdot 2 \pi\left[\left\{l_{0}+K^{2}\left(l_{3}+3 l_{4}\right)\right\} \alpha+K\left(l_{1}+l_{2}\right)\right] .
\end{aligned}
$$

At the aerodynamic center, the corresponding moment coefficient should be independent of the angle of attack $\alpha$. From Eq. (64), this condition gives the distance $d$ of aerodynamic center back of the leading edge as

$$
\frac{d}{c}=\frac{1}{2}-\frac{1}{4} \frac{m_{0}+K^{2}\left(2 m_{3}+4 m_{4}\right)}{l_{0}+K^{2}\left(l_{3}+3 l_{4}\right)} .
$$

Then the moment about the aerodynamic center is given by the following coefficient:

$$
C_{M_{\text {a.c. }}}=\frac{\pi K}{2}\left[\left(m_{1}+m_{2}\right)-\frac{m_{0}+K^{2}\left(2 m_{3}+4 m_{4}\right)}{l_{0}+K^{2}\left(l_{3}+3 l_{4}\right)}\left(l_{1}+l_{2}\right)\right] .
$$

The numerical values of $d / c$ and $C_{M_{\text {a.c. }}}$ calculated from Eqs. (65) and (66) are plotted in Figs. 7 and 8 against the thickness ratio $\delta$ for different values of $K$. 